\title{
VALORES BIOQUÍMICOS SANGUÍNEOS EN HEMBRAS BRAHMAN BAJO CONDICIONES DE PASTOREO ${ }^{1}$
}

\author{
NESTOR ALONSO VILLA ${ }^{2}$, ALEJANDRO CEBALLOS ${ }^{3}$, DOUGLAS CERON ${ }^{2}$ y CARLOS ALBERTO SERNA ${ }^{2}$
}

\begin{abstract}
RESUMEN - Con el objeto de establecer un rango de referencia para diferentes metabolitos en hembras bovinas $B$. indicus, se tomaron 5-10 $\mathrm{mL}$ de suero sanguíneo a 21 hembras bovinas Brahman registradas en el departamento de Caldas, Colombia. Se determinó la concentración de los diferentes metabolitos que forman parte de un perfil metabólico estándar, como ß-hidroxibutirato, proteína total, albúmina, globulinas, urea, aspartato aminotransferasa (AST), calcio y magnesio. Los resultados fueron analizados mediante la obtención del rango, promedio y desviación estándar (DE). El intervalo de referencia se estableció en el $95 \%(\mathrm{x} \pm 2 \mathrm{DE})$. El promedio y la DE obtenidos para cada metabolito fueron: ß-hidroxibutirato $0,34 \pm 0,15 \mathrm{mmol} / \mathrm{L}$; proteína total $81 \pm 9 \mathrm{~g} / \mathrm{L}$; albúmina $41 \pm 3 \mathrm{~g} / \mathrm{L}$; globulinas $40 \pm 10 \mathrm{~g} / \mathrm{L}$; urea $3,31 \pm 1,43 \mathrm{mmol} / \mathrm{L}$; AST $158 \pm 34 \mathrm{U} / \mathrm{L} ; \mathrm{Ca} 2,50 \pm 0,21 \mathrm{mmol} / \mathrm{L}$ y Mg 1,40 $\pm 0,44 \mathrm{mmol} / \mathrm{L}$. Se observó que las alteraciones más frecuentes fueron aumento en la concentración de butiratos y globulinas y aumento en la actividad de AST. Según los resultados obtenidos, se puede señalar que los valores encontrados para la concentración de diferentes metabolitos séricos en hembras Brahman mantenidas en pastoreo, son similares a los valores descritos para bovinos B. taurus.
\end{abstract}

Términos para índice: bovinos, bioquímica clínica, perfil metabólico.

\section{CLINICAL BIOCHEMISTRY VALUES IN SERUM FROM GRAZING BRAHMAN COWS}

\begin{abstract}
This study was designed in order to get the reference values for the concentration of different metabolites included in a standard metabolic profile for grazing Brahman cattle (B. indicus) from Caldas, Colombia. Serum samples $(5-10 \mathrm{~mL})$ were taken from 21 healthy grazing Brahman cows to analyse $\beta$-hydroxybutyrate, total protein, albumin, globulines, urea, aspartate aminotransferase (AST), calcium and magnesium. The results were analyzed by calculating range, mean and standard deviation (SD). The reference interval was set at 95\% ( $x \pm 2 \mathrm{SD})$. The mean and SD obtained for the concentration of the different metabolites were: $\beta$-hydroxybutyrate $0.34 \pm 0.15 \mathrm{mmol} / \mathrm{L}$; total protein $81 \pm 9 \mathrm{~g} / \mathrm{L}$; albumin $41 \pm 3 \mathrm{~g} / \mathrm{L}$; globulines $40 \pm 10 \mathrm{~g} / \mathrm{L}$; urea $3.31 \pm 1.43 \mathrm{mmol} / \mathrm{L}$; AST $158 \pm 34 \mathrm{U} / \mathrm{L}$; $\mathrm{Ca} 2.50 \pm 0.21 \mathrm{mmol} / \mathrm{L}$, and $\mathrm{Mg} 1.40 \pm 0.44 \mathrm{mmol} / \mathrm{L}$. The increasing of $\beta$-hydroxybutyrates and globulines, and a high activity of AST were the most frequent alterations. The serum biochemistry values for grazing $B$. indicus cattle are similar to the described values for $B$. taurus.
\end{abstract}

Index terms: cattle, clinical biochemistry, metabolic profile.

\section{INTRODUCCIÓN}

Un perfil metabólico (PM) es un conjunto de determinaciones de laboratorio que permiten la

1 Aceptado para publicación en 25 de noviembre de 1998.

2 Méd. Vet. Zoot., M.Sc., Facultad de Ciencias Agropecuarias, Universidad de Caldas, Manizales, Colombia. E-mail: navilla@hotmail.com

${ }^{3}$ Méd. Vet. Zoot., M.Sc., Escuela de Medicina Veterinaria, Universidad Católica de Temuco, Temuco, Chile. E-mail: aceballo@uctem.cl caracterización de un individuo o grupo de ellos y tienen por objeto aportar una ayuda paraclínica para estudiar la naturaleza de los trastornos metabólicos (Oyarzún, 1997). En la realización de un PM se determinan los diferentes metabolitos sanguíneos relacionados con el estado funcional de las vías metabólicas (biotransformación), las que están determinadas por el consumo de nutrientes al seguir diferentes vías después de su ingestión en el organismo; el estado de estas vías puede verse afectado por los desbalances en el ingreso, transformación o egreso de los ingredientes de la 
ración consumida por los animales (Payne et al., 1970; Payne \& Payne, 1987).

Los análisis realizados en un PM estándar son diversos incluyéndose varios metabolitos; en general, se pretende evaluar el estado de las vías metabólicas que guardan relación con el aporte de proteína, energía y minerales. El PM estándar desarrollado por el Laboratorio de Patología Clínica Veterinaria de la Universidad Austral de Chile incluye la determinación de hemoglobina, ß-hidroxibutiratos, urea, proteínas totales, albúmina, globulinas, calcio, fósforo, magnesio y aspartato aminotransferasa (AST; EC 2.6.1.1), lo que permite evaluar el estado metabólico de las vías metabólicas descritas, así como el funcionamiento hepático (Wittwer et al., 1987; Oyarzún, 1997).

En el PM se analizan las muestras sanguíneas tomadas a un grupo representativo de animales, expresándose los resultados en términos de promedio y desvíos para cada variable analizada; posteriormente, se establecen las comparaciones para cada metabolito según los valores reportados como referencia para la población y así, puede evaluarse el estado nutricional, metabólico y de salud del rebaño (Rowlands \& Pocock, 1976).

Desde la aparición de los PM en la década de 1970 se han reportado valores de referencia para bovinos de diferentes razas pertenecientes a la especie Bos taurus y mantenidos en diferentes zonas templadas (Payne et al., 1970; Collins, 1979; Wittwer \& Contreras, 1980; Roussel et al., 1982; Ward et al., 1995; Oyarzún, 1997). Para bovinos pertenecientes a la especie $B$. indicus los valores de referencia señalados por la literatura son escasos en comparación con los reportes para B. taurus; además, si se considera que la mayor parte de la población de ganados índicos está en zonas tropicales, los valores reportados son aún más escasos. No obstante, se han señalado algunos valores en diferentes investigaciones; así, se han descrito valores para la concentración sérica de minerales (Awolaja et al., 1997) y algunos metabolitos en ganado B. indicus mantenido en pastoreo en diferentes regiones tropicales (Sawadogo et al., 1991; Ortolani, 1995).

En consideración a lo anterior, el presente trabajo tiene por objeto establecer un rango de referencia para los metabolitos analizados en un perfil metabólico estándar para bovinos Brahman (B. indicus) mantenidos bajo condiciones de pastoreo.

\section{MATERIAL Y MÉTODOS}

En el departamento de Caldas, Colombia se seleccionó un predio según su masa ganadera y tipo de alimentación del rebaño. Las características climáticas de la zona están determinadas por una zona de convergencia intertropical caracterizadas por dos épocas de verano y dos épocas de lluvia, donde la altitud era 985 metros sobre el nivel del mar con una temperatura promedio de $25^{\circ} \mathrm{C}$, una humedad relativa del $75 \%$ y una pluviosidad de 2020 milímetros por año.

El predio tenía una extensión de 767 hectáreas con una topografía caracterizada por ser ondulada y con suelos franco-arcillosos. El área destinada a la ganadería (511 hectáreas) estaba compuesta por pradera natural no fertilizada (Paspalum spp.) y pasto india (Panicum maximum), puntero (Hyparrhenia rufa) y pangola (Digitaria decumbens) no fertilizados. Los potreros se manejaban bajo un sistema de rotación alterna con una carga animal de 2,5 animales/hectárea. El control de malezas se realizaba mediante prácticas culturales manuales y mecánicas.

Los animales estaban mantenidos bajo un sistema de manejo semi-intensivo con partos durante todo el año, la alimentación estaba basada en la utilización de la pradera y suplementación ad libitum con sales minerales. El sistema de servicio en las hembras era por inseminación artificial, empleando un toro para servir sólo aquellas hembras no gestantes después de dos servicios por inseminación.

En el mes de abril de 1997 se seleccionaron 21 hembras adultas registradas de la raza Brahman clínicamente sanas, a las que se tomaron $5-10 \mathrm{~mL}$ de sangre sin anticoagulante mediante venopunción coccígea empleando el sistema de tubos al vacío. Las muestras fueron remitidas dentro de 24 horas a un laboratorio de patología clínica particular donde se realizaron las determinaciones (Cuadro 1).

Las muestras fueron centrifugadas para separar el coágulo, posteriormente se determinaron los metabolitos señalados en el PM estándar, para lo cual se emplearon las técnicas descritas en el Cuadro 1. La exactitud y precisión para cada una de las técnicas se controlaron mediante la utilización de un suero con valores conocidos (Lyotrol. Laboratorio BioMérieux, Francia).

El tipo de distribución de los datos se evaluó mediante la prueba de normalidad descrita por Pearson \& Stephens (1964). Se obtuvieron el rango y las estimadas promedio 
( $\bar{X}$ ) y desviación estándar (DE) (Weisbrot, 1985). El rango de referencia, determinado por un intervalo de confianza del $95 \%$, para cada metabolito cuya distribución de valores era paramétrica se obtuvo mediante la metodología descrita por Bermes Junior et al. (1976) y Weisbrot (1985), se empleó la siguiente ecuación:

$I C_{\bar{\chi}}=\bar{\chi} \pm 2 \sigma_{\bar{\chi}}$.

Se obtuvieron los percentiles 2,5 y 97,5 para las variables cuya distribución era no paramétrica (Kaneko et al., 1997). Se presenta la frecuencia de individuos que mostraron valores menores o superiores a los límites inferior y superior según el rango obtenido.

\section{RESULTADOS Y DISCUSIÓN}

Los valores promedio, la desviación estándar y el intervalo de confianza del 95\% (x $\pm 2 \mathrm{DE})$ para B-hidroxibutirato, proteína, albúmina, globulinas, urea, aspartato aminotransferasa, Ca y $\mathrm{Mg}$, obtenidos en el análisis de 21 perfiles metabólicos en hembras
Brahman registradas se presentan en el Cuadro 2. Las alteraciones más frecuentemente encontradas en los individuos analizados fueron aumento en la concentración de butiratos $(4,7 \%)$ y globulinas $(4,7 \%)$ y aumento en la actividad de AST $(9,5 \%)$ (Fig. 1).

Los valores que se obtuvieron en este estudio (Cuadro 2) son similares a los que han sido reportados para algunas razas bovinas pertenecientes a la especie B. taurus (Boehringer Mannheim GmbH, 1981; Kaneko et al., 1997). El intervalo de confianza se obtuvo tomando como base un mínimo de 20 determinaciones para el mismo metabolito en animales diferentes y clínicamente sanos, lo que permite tener una aproximación representativa e inicial de los valores para diferentes metabolitos en la población de interés. No obstante, Bermes Junior et al. (1976) señalan que sería deseable tener un número de datos cercano a 100, lo que en algunas oportunidades no es posible; por lo tanto, el manejo

CUADRO 1. Variables sanguíneas analizadas, unidades, método y muestra utilizada.

\begin{tabular}{llll}
\hline Variable & Unidad & Metodo & Muestra \\
\hline ß-hidroxibutirato & $\mathrm{mmol} / \mathrm{L}$ & Cinético ultravioleta & Suero \\
Proteína total & $\mathrm{g} / \mathrm{L}$ & Biuret & Suero \\
Albúmina & $\mathrm{g} / \mathrm{L}$ & Verde de bromocresol & Suero \\
Globulinas & $\mathrm{g} / \mathrm{L}$ & Diferencia: proteína total - albúmina & Suero \\
Urea & $\mathrm{mmol} / \mathrm{L}$ & Azul de indofenol & Suero \\
Aspartato aminotransferasa (AST; EC 2.6.1.1) & $\mathrm{U} / \mathrm{L}$ & Cinético (DGKC, 37 C) & Suero \\
Calcio & $\mathrm{mmol} / \mathrm{L}$ & Colorimétrico, azul de metiltimol & Suero \\
Magnesio & $\mathrm{mmol} / \mathrm{L}$ & Colorimétrico, magnesio calmagita & Suero \\
\hline
\end{tabular}

CUADRO 2. Promedio (x), desviación estándar (DE) y rango (IC) para los metabolitos analizados en un grupo de hembras Brahman registradas en el departamento de Caldas, Colombia.

\begin{tabular}{|c|c|c|c|c|}
\hline Variable & Unidad & $\mathrm{x}$ & $\mathrm{DE}$ & $\mathrm{IC}^{1}$ \\
\hline B-hidroxibutirato & $\mathrm{mmol} / \mathrm{L}$ & 0,34 & 0,15 & $0,04-0,64$ \\
\hline Proteína total & $\mathrm{g} / \mathrm{L}$ & 81 & 9 & $63-99$ \\
\hline Albúmina & $\mathrm{g} / \mathrm{L}$ & 41 & 3 & $35-47$ \\
\hline Globulinas & $\mathrm{g} / \mathrm{L}$ & 40 & 10 & $20-60$ \\
\hline Urea & $\mathrm{mmol} / \mathrm{L}$ & 3,31 & 1,43 & $0,50-6,17$ \\
\hline Aspartato aminotransferasa (AST; EC 2.6.1.1) & $\mathrm{U} / \mathrm{L}$ & 158 & 34 & $90-226$ \\
\hline Calcio & $\mathrm{mmol} / \mathrm{L}$ & 2,50 & 0,21 & $2,08-2,92$ \\
\hline Magnesio & $\mathrm{mmol} / \mathrm{L}$ & 1,40 & 0,44 & $0,52-2,28$ \\
\hline
\end{tabular}

${ }^{1}$ Intervalo de confianza (95\%). 
de los datos para la obtención del intervalo de confianza va a depender del método estadístico utilizado.

En la población objeto del estudio se pueden producir variaciones en los resultados, las que están dadas por factores como sexo, raza, edad, estado productivo, etc. (Wittwer \& Böhmwald, 1986). En este estudio se analizó un grupo de hembras clínicamente sanas y mantenidas bajo el mismo sistema productivo, pero no se hicieron determinaciones para establecer las diferencias que pudieran presentarse con otros animales de diferentes edades o con machos, ya que el objeto era realizar una aproximación inicial a los valores en bovinos $B$. indicus en Colombia.

Los valores obtenidos son similares a los obtenidos en otros estudios donde se ha realizado este tipo de determinaciones en bovinos $B$. indicus; no obstante la concentración promedia para urea es $0,84 \mathrm{mmol} / \mathrm{L}$ mayor que el valor descrito por Sawadogo et al. (1991) para animales mantenidos en pastoreo, mientras que el promedio observado para la concentración de calcio es inferior en $0,06 \mathrm{mmol} / \mathrm{L}$ al reportado por los mismos autores. El promedio en la concentración de magnesio se encontraba $0,09 \mathrm{mmol} / \mathrm{L}$ por encima del valor señalado por Ortolani (1995) en bovinos cebú.

Los resultados encontrados en este grupo de hembras es difícil compararlos con los valores señalados por la literatura como referencia, ya que

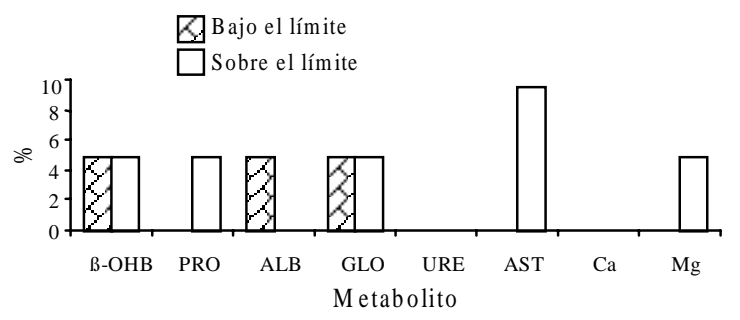

FIG. 1. Frecuencia de individuos con valores bajo y sobre el intervalo de confianza del $95 \%$ para los metabolitos analizados en un grupo de hembras Brahman registradas en el departamento de Caldas, Colombia. los reportes son de estudios realizados con otras especies o razas bovinas y bajo otras condiciones de manejo; además, aunque los valores reportados son coincidentes con los observados, hay mucha disparidad en el intervalo de confianza considerado normal para cada metabolito; hecho que se había observado con anterioridad en otros estudios de naturaleza similar (Wittwer et al., 1987). Igualmente, cabe señalar que la literatura relacionada con los valores para diferentes metabolitos bioquímicos sanguíneos en ganado $B$. indicus es escasa.

Las alteraciones observadas con mayor frecuencia en este estudio según los valores observados fueron aumento en la concentración de butiratos y globulinas, y aumento en la actividad de AST (Fig. 1).

Se ha señalado que la concentración de butiratos debe ser inferior a $0,9 \mathrm{mmol} / \mathrm{L}$ (Ward et al., 1995), no obstante, en este estudio se ha considerado que un valor normal debe ser inferior a $0,46 \mathrm{mmol} / \mathrm{L}$ (Kaneko et al., 1997); el aumento de la concentración de butiratos está relacionado con desbalances energéticos en la dieta del animal, pero el hallazgo de valores anormales en un individuo no es un indicador de la presencia de alteraciones metabólicas, lo que no ocurre cuando se observan varios valores alterados dentro de un mismo grupo de animales (Ward et al., 1995).

El aumento en la concentración de globulinas ocurre cuando hay presencia de procesos infecciosos en el organismo (Duncan et al., 1994), pudiendo señalarse también que la aparición de un valor alterado no indica que el grupo estudiado se encuentre cursando con problemas de esta índole.

La actividad sanguínea de AST se encontró elevada en un 9,5\% de los individuos estudiados. Los valores alterados para la actividad de esta enzima son compatibles con la presencia de lesiones en la musculatura estriada esquelética o cardíaca y hepáticas, siendo un indicador de daño celular altamente sensible pero poco específico (Duncan et al., 1994), cabe señalar que los análisis enzimáticos se caracterizan por presentar una gran variación en los resultados, lo que significa que pocos valores alterados dentro de un mismo grupo de animales puede no representar la situación real de la población (Ward et al., 1995). 


\section{CONCLUSIÓN}

Los valores obtenidos para la concentración sérica de diferentes metabolitos en hembras bovinas B. indicus mantenidas en pastoreo, son similares a los descritos para bovinos B. taurus, lo que permite en un momento dado y mientras no se tengan los propios valores de referencia, emplear los valores descritos en $B$. taurus para establecer comparaciones.

\section{REFERENCIAS}

AWOLAJA, O.A.; ANTIA, R.E.; OYEJIDE, A. Trace element levels in plasma/serum and erythrocytes of Keteku and White Fulani cattle. Tropical Animal Health and Production, v.29, p.2-6, 1997.

BERMES JUNIOR, E.W.; ERVITI, V.; FORMAN, D.T. Statistics, normal values, and quality control. In: TIETY, N.W. (Ed.). Fundamentals of clinical chemistry. Philadelphia: W.B. Saunders Co, 1976.

B OEHRINGER MANNHEIM GMBH.Laboruntersuchungen für die Diagnose und Verlaufskontrolle in der Veterinärmedizin. Mannheim, 1981. 230p.

COLLINS, J.D. Metabolic profile tests for dairy cattle. Irish Veterinary Journal, v.33, n.2, p.26-31, Feb. 1979.

DUNCAN, J.R.; PRASSE, K.W.; MAHAFFEY, E.A Veterinary laboratory medicine. Ames: Iowa State University Press, 1994. 300p.

KANEKO, J.J.; HARVEY, J.W.; BRUSS, M.L. Clinical biochemistry of domestic animals. San Diego: Academic Press, 1997. 932p.

ORTOLANI, E.L. Aspectos clínicos, epidemiológicos e terapêuticos da hipocalcemia de vacas leiteiras. Arquivos Brasileiros de Medicina Veterinária e Zootecnia, v.47, n.6, p.799-808, 1995.

OYARZÚN, J.L. Análisis de los resultados de perfiles metabólicos obtenidos de rebaños lecheros en el sur de Chile 1986-1996. Valdivia: Universidad Austral de Chile, Facultad de Ciencias Veterinarias, 1997. 34p. Tesis Licenciado.
PAYNE, J.M.; DEW, S.M.; MANSTON, R.; FAULKS, M. The use of metabolic profile test in dairy herds. The Veterinary Record, v.87, p.150-158, Aug. 1970.

PAYNE, J.M.; PAYNE, S. The metabolic profile test. Oxford: Oxford University Press, 1987. 179p.

PEARSON, E.S.; STEPHENS, M.A. The ratio of range to standard deviation in the same normal sample. Biometrika, v.51, p.484-487, 1964.

ROUSSEL, J.D.; SEYBT, S.H.; TOUPS, G. Metabolic profile testing for Jersey cows in Louisiana: Reference values. The American Journal of Veterinary Research, v.43, n.6, p.1075-1077, Jun. 1982.

ROWLANDS, G.J.; POCOCK, R.M. Statistical basis of the Compton metabolic profile test. The Veterinary Record, v.98, p.333-338, Apr. 1976.

SAWADOGO, G.J.; OUMAROU, A.A.; SENE, M.; DIOP, M. Effects of poor pasture conditions and type of feeding on some biochemical values of Gobra zebu in Senegal. British Veterinary Journal, v.147, p.538-544, 1991.

WARD, W.R.; MURRAY, R.D.; WHITE, A.R.; REES, E.M. The use of blood biochemistry for determining the nutritional status of dairy cows. In: GARNSWORTHY, P.C.; COLE, D.J.A. (Eds.). Recent advances in animal nutrition. Nottingham: Nottingham University Press, 1995. $274 \mathrm{p}$.

WEISBROT, I.M. Statistics for the clinical laboratory. Philadelphia: J.B. Lippincott Company, 1985. 198p.

WITTWER, F.; BÖHMWALD, H. Manual de patología clínica veterinaria. Valdivia: Universidad Austral de Chile, 1986. 167p.

WITTWER, F.; BÖHMWALD, H.; CONTRERAS, P.A.; FILOZA, J. Análisis de los resultados de perfiles metabólicos obtenidos en rebaños lecheros en Chile. Archivos de Medicina Veterinaria, v.19, n.2, p.35-45. Jul./Dic. 1987.

WITTWER, F.; CONTRERAS, P.A. Empleo de los perfiles metabólicos en el sur de Chile. Archivos de Medicina Veterinaria, v.12, n.2, p.221-228. Jul/ Dic. 1980. 